\title{
INVESTIGATIONS OF DEFECT CENTRES IN ALKALI HALIDES DOPED WITH TRIVALENT CATIONS. PART 1: BISMUTH IN NaCl, $\mathrm{KCl}$ AND RbCl CRYSTALS
}

\author{
M. SUSZYŃSKA \\ W. Trzebiatowski Institute of Low Temperature and Structure Research \\ Polish Academy of Sciences \\ Pl. Katedralny 1, 50-950 Wrocław, Poland \\ and R. Capelletti \\ Physics Department, Parma University, 43100 Parma, Italy \\ Dedicated to Professor Dr. Julian Auleytner \\ on the occasion of his 70th birthday
}

(Received July 9, 1992)

A correlated set of experiments was performed with a view to study the effect of trivalent bismuth upon some properties of $\mathrm{NaCl}, \mathrm{KCl}$ and $\mathrm{RbCl}$ crystals. For $\mathrm{NaCl}$ the effect of $\mathrm{BiO}^{+}$ions was also investigated. It was stated that: 1 . The hydrolytic properties of bismuth are responsible for the formation of $\mathrm{BiO}^{+}$centres also in crystals doped with $\mathrm{Bi}^{3+}$ ions. 2. During all experiments performed, the dopant remains in the form of trivalent cations. 3. Optical absorption spectra of bismuth are similar to those characteristic of other heavy metal ions with the $s^{2}$ electronic configuration. 4 . The charge excess of $\mathrm{Bi}^{3+}$ ions is compensated by cation vacancies whereas for $\mathrm{BiO}^{+}$ ions the preferential bonding between $\mathrm{Bi}^{3+}$ and $\mathrm{O}^{2-}$ serves for the charge compensation.

PACS numbers: $77.40 .+i$

\section{Introduction}

Many papers have reported the effect of impurities upon different physical properties of alkali halide $(\mathrm{AH})$ crystals. Most of these works dealt with divalent cations $\left(\mathrm{Me}^{2+}\right)$ present in sodium and potassium chlorides. Only few experiments 
have been performed on crystals doped with trivalent impurities $\left(\mathrm{Me}^{3+}\right)$. The significance of these works relates, among others, with a renewed interest in spectroscopic properties of simple salts doped with elements with the $d$ - and $f$-electrons.

Dielectric properties of trivalent cations in monovalent ionic crystals, like alkali- and silver halides, were up to now studied by various authors [1-5]. According to Kunze et al. [5] such ions as $\mathrm{Ti}^{3+}, \mathrm{Cr}^{3+}$ and $\mathrm{V}^{3+}$ enter substitutionally the monovalent lattice of $\mathrm{AgCl}$ and at sufficiently low temperature are completely associated with two cation vacancies. These complexes are able to reorient by a thermally activated jump of one of the species involved. Like the impurity-vacancy (I.V.) dipoles in AH: $\mathrm{Me}^{2+}$ systems, the pertinent defects in the $\mathrm{Me}^{3+}$ doped crystals have been assigned impurity-vacancy-vacancy (I.V.V.) dipoles.

To obtain further information about the composed character of the mentioned defects and their configuration within the matrix, about the number and distribution of the excess charge compensating cation vacancies etc., bismuth doped $\mathrm{NaCl}, \mathrm{KCl}$ and $\mathrm{RbCl}$ crystals (Part 1 ) and chromium doped $\mathrm{NaCl}$ crystals (Part 2) were studied by means of ionic thermocurrent (ITC) technique. These measurements were complemented by investigations of some spectroscopical, electric and mechanical characteristics. It should be noted that the ionic radius of these trivalent cations is smaller than that of the host cations and the geometrical situation in the $\mathrm{AH}: \mathrm{Me}^{3+}$ systems studied resembles that of silver halides doped with trivalent impurities [5].

\section{Experimental details}

\subsection{Origin of crystals and the dispersion state of the dopant}

The crystals were grown by Dr M. Lebl from Scientific Service in Prague (Czecho-Slovakia) according to a modified Bridgman method [6]; for basic characteristics of the samples see Table I. As received (AR) crystals were used mainly. To modify the state of aggregation of the dopant, AR samples were annealed at $873 \mathrm{~K}$ for various times; the multiply of 30 minutes was employed. After each period of annealing, the hot sample was dropped onto a copper plate at room temperature (RT). This procedure defines the solution treated (ST) state of impurities. In some instances the effect of uniaxial compression (along a $\langle 100\rangle$ direction) was investigated. The deformation was realized in a self-made device with load calibrated against the 1112 INSTRON machine working at a strain rate equal to $3 \times 10^{-4} \mathrm{~s}^{-1}$.

\subsection{Valency of bismuth}

When bismuth enters the crystal lattice in a trivalent state, no paramagnetism is expected as the outer electronic configuration is $6 s^{2}$, whereas if it is in the divalent state, associated paramagnetic behaviour occurs. EPR spectroscopy was used to characterize and to verify the valency of the dopant in both as received as well as thermally and mechanically pretreated samples. The spectra were taken at RT and liquid nitrogen temperature (LNT) with the VARIAN-V-4502 spectrometer. Independently of the crystal matrix, type and concentration of the dopant 


\section{TABLE I}

Basic characteristics of the crystals.

\begin{tabular}{c|r|r|r}
\hline \hline System & Mark & $c$ [mppm] & $\alpha$ cm $\left.^{-1}\right]^{* *}$ \\
\hline NaCl:Bi(3+) & $\mathrm{D} .1$ & 50 & 0.38 \\
& 2 & 100 & 1.52 \\
& 3 & 1000 & 0.81 \\
& 4 & 1000 & 5.01 \\
& 6 & 5000 & 2.97 \\
$\mathrm{NaCl}: \mathrm{BiO}(+)$ & $\mathrm{E} .2$ & 50 & 1.12 \\
& 4 & 100 & 0.52 \\
& 5 & 1000 & 8.01 \\
& 6 & 2000 & 14.82 \\
& 7 & 5000 & 1.62 \\
& 8 & 10000 & 17.62 \\
& 9 & 1000 & 2.16 \\
& 10 & 800 & 4.61 \\
$\mathrm{KCl}: \mathrm{Bi}(3+)$ & $\mathrm{M} .1$ & 1000 & 3.90 \\
& 2 & 1000 & 20.71 \\
$\mathrm{RbCl}: \mathrm{Bi}(3+)$ & $\mathrm{R} .1$ & 1000 & - \\
\hline
\end{tabular}

*Dopant concentration in the melt.

**Absorption coefficient of the high-energy band $\alpha^{C} / \alpha^{A} \cong 4.5,4.3$ and 7 for $\mathrm{E}, \mathrm{D}$ and $M$ crystals respectively.

and the pretreatment of samples, no EPR signal related with divalent bismuth was registered.

Preliminary studies of the mechanical characteristics permitted also to exclude the presence of monovalent bismuth ions, in the $\mathrm{NaCl}$ crystals at least. Figure 1 shows the concentration dependence of Vickers microhardness taken at $0.1 \mathrm{~N}$ for $\mathrm{Bi}^{3+}$ - (curve $a$ ) and $\mathrm{BiO}^{+}$- (curve $b$ ) doped AR samples. The impurity induced strengthening of slightly doped crystals is followed by a relative softening of samples with the largest amount of the dopant. Both phenomena are explainable in the frame of elastic interactions between moving dislocations and tetragonal strain fields related with the impurity obstacles [7, 8]. For monovalent impurities, located substitutionally in the $\mathrm{NaCl}$ matrix, the local symmetry of the induced. strain fields should remain cubic, presenting much less effective obstacles for the moving dislocations. 


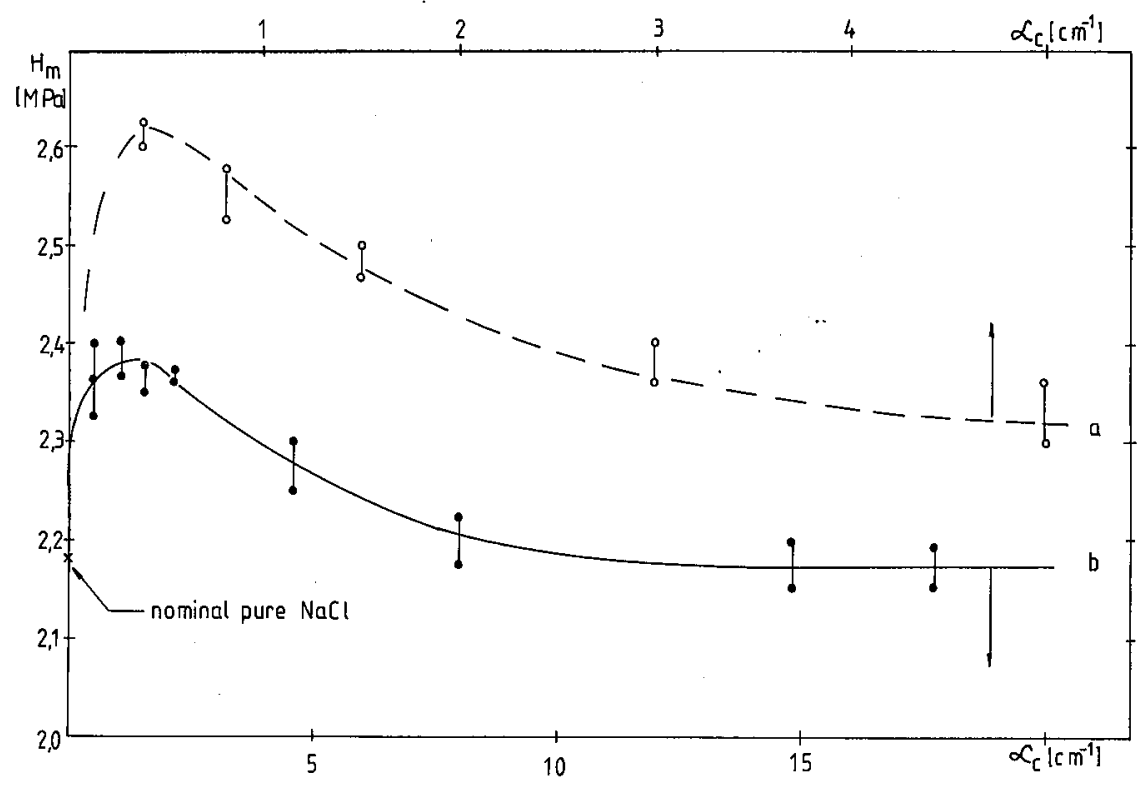

Fig. 1. Concentration dependences of the Vickers microhardness for bismuth doped $\mathrm{NaCl}$ crystals; curves $a$ and $b$ concern $\mathrm{Bi}(3+)$ and $\mathrm{BiO}(+)$ doped crystals, respectively; the concentration is represented by the absorption coefficient value of the $C$ band.

\subsection{Other measurements performed}

Optical absorption spectra were measured between 195 and $800 \mathrm{~nm}$ by means of a SPECORD (Zeiss) spectrophotometer. Conductivity data were obtained by taking the resistance of samples subjected to $100 \mathrm{~V}$ of dc; a GENERAL RADIO 1621 bridge was used for these measurements. To avoid polarization effects the dc voltage was applied for a short time to crystal faces coated with colloidal graphite. Dielectric relaxation phenomena were studied by measuring thermally stimulated depolarization currents (ITC) according to the procedure developed by Bucci et al. [9]. The measurements were usually performed in an extended range of testing temperature (LNT-373 K). For plastically deformed samples the measurement arrangement has been described elsewhere [10].

\section{Results and discussion}

\subsection{Analysis of the absorption dala}

\subsubsection{Absorption spectra}

Figures 2 and 3 show some examples of the optical absorption spectra of bismuth doped crystals, and Table II lists the positions of bands detected at RT and LNT. Three bands $(A, B, C)$ were usually observed, from which the $C$ band exhibits a more or less distinct structure when measured at LNT; these findings are 

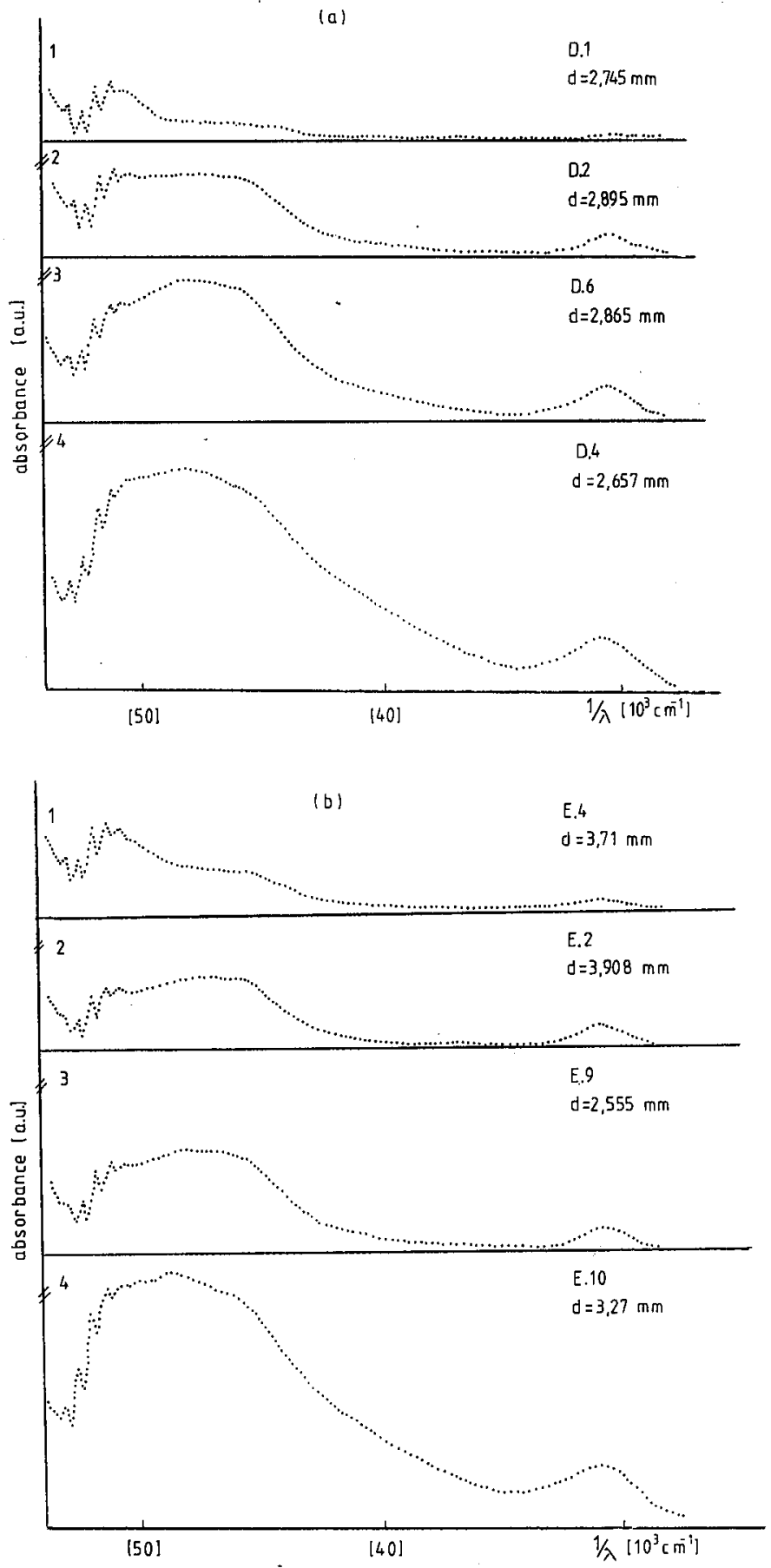

Fig. 2. Optical absorption spectra of $\mathrm{NaCl}$ crystals doped with various amounts of $\mathrm{Bi}^{3+}(\mathrm{a})$ and $\mathrm{BiO}^{+}(\mathrm{b})$ ions. 

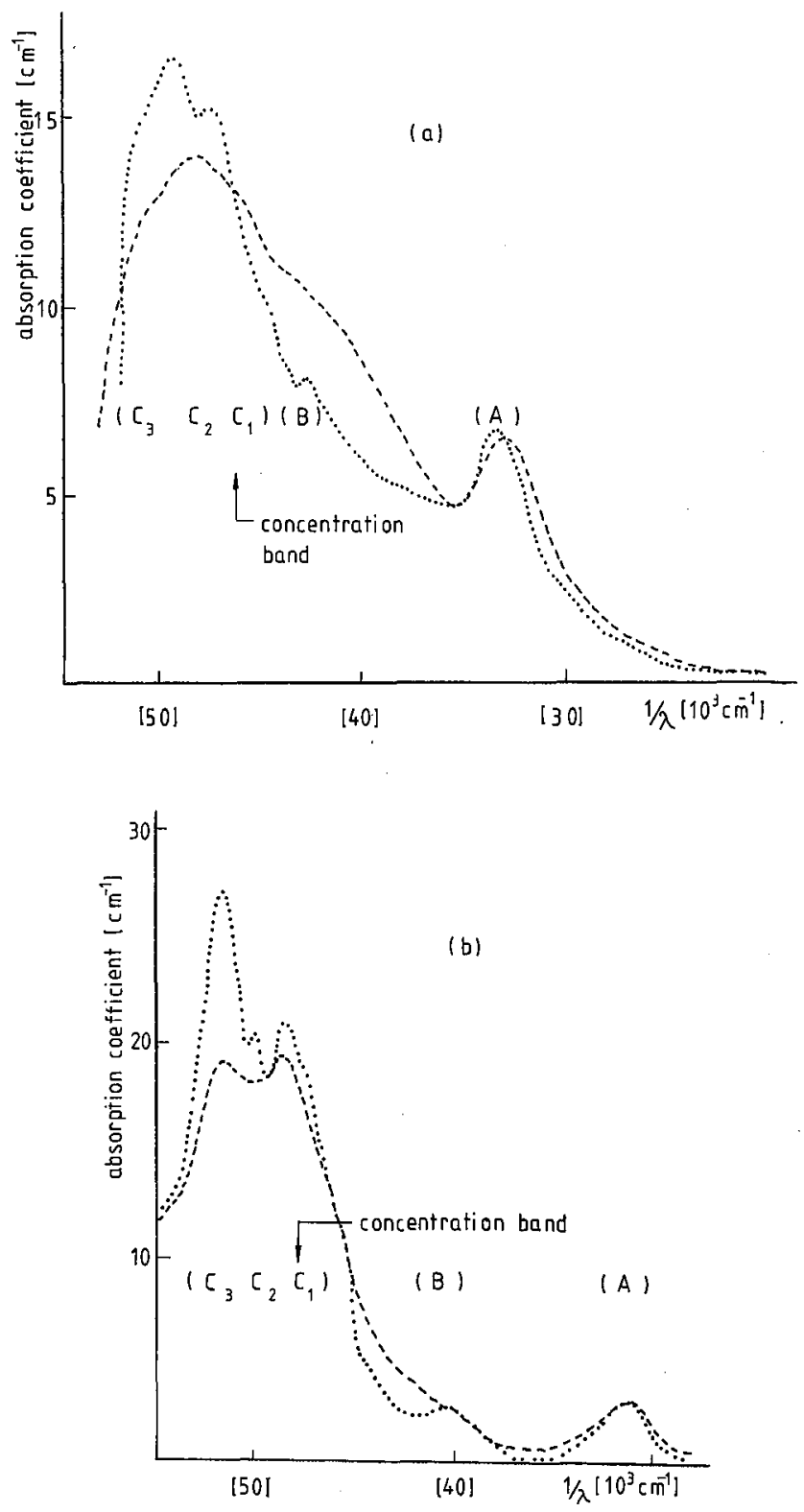

Fig. 3. Optical absorption of E.6 (a) and M.2 (b) crystals measured at RT ( $($ ) and LNT (•); for the first spectrum the $\alpha^{C} / \alpha^{A}$ ratio is equal only to 2.18 .

essentially consistent with the literature data. Some pecularities registered were as follows.

For both types of the dopant in $\mathrm{NaCl}$ crystals the spectra exhibit some dif- 
TABLE II

Positions of the absorption bands induced by bismuth in the alkali halide crystals.

\begin{tabular}{c|c|c|c|c}
\hline \hline \multirow{2}{*}{ System } & \multicolumn{2}{|c|}{$A$-band } & \multirow{2}{*}{$B$-band } & $C_{1} \div C_{2}$ \\
\cline { 2 - 5 } & RT & LNT & LNT & LNT \\
\hline D & 328 & 325 & 240 & $195 \div 212$ \\
E & 320 & 320 & - & $195 \div 213$ \\
M & 330 & 325 & 244 & $201 \div 212$
\end{tabular}

ferences with respect to the testing temperature. At LNT, for instance, a shift of the position of the lowest-energy band $(A)$ towards shorter wavelengths and the absence of the next band $(B)$ were detected for crystals nominally doped with $\mathrm{Bi}^{3+}$ and $\mathrm{BiO}^{+}$ions, respectively. For both dopants the bands observed at $195 \mathrm{~nm}$ are probably related with the presence of some oxygen-containing centres. The preferential bonding between $\mathrm{Bi}^{3+}$ and $\mathrm{O}^{2-}$ assures the excess charge compensation in $\mathrm{BiO}^{+}$centres. Annealing of solution treated samples at temperatures up to about $573 \mathrm{~K}$ yields a decrease in the intensity of the $A$ and $C$ bands. The ratio of the absorption coefficients $\alpha^{A} / \alpha^{C}$ is only slightly larger for the $\mathrm{BiO}^{+}$doped samples. Similar behaviour of both bands speaks in favour of the fact that they are related with the same type of optically active centres.

For $\mathrm{KCl}: \mathrm{Bi}^{3+}$ crystals the $B$ band as well as the structure of the $C$ band disappear when the testing temperature increases above $80 \mathrm{~K}$. Moreover, the solution treatment induces a shift of the $A$ band towards longer wavelengths.

For $\mathrm{RbCl}: \mathrm{Bi}^{3+}$ crystals no optical absorption was detected all over the UV spectral range for samples as thick as about $2.5 \mathrm{~mm}$; for thicker samples an absorption trace was registered between 280 and $330 \mathrm{~nm}$. These facts are probably consequences of an extremely low solubility limit of trivalent bismuth in the rubidium salts.

\subsubsection{Determination of dopant concentration}

General features of the absorption spectra obtained are similar to those characteristic of other alkali halides containing small amount of heavy metal ions with the mercury-like $s^{2}$.configuration, see for instance the work by Fukuda [11]. Thus, the pertinent absorption bands should correspond to the following transitions:

$$
{ }^{1} S_{0} \rightarrow{ }^{1} P_{1} \text { and }{ }^{1} S_{0} \rightarrow{ }^{3} P_{0},{ }^{1} S_{0} \rightarrow{ }^{3} P_{1},{ }^{1} S_{0} \rightarrow{ }^{3} P_{2}
$$

in the impurity cations, whose levels are perturbed by the crystal field. Exploiting this similarity, bismuth concentration was calculated from the Smakula equation describing the relationship between the number $N$ of optically active centres per $\mathrm{cm}^{3}$ and the absorption coefficient $\boldsymbol{a}^{C}$ of the $C$ band. Taking for the parameters in this equation: 0.5 for the oscillator strength $f^{C}$ [11], 1.8 for refractive index $n(\mathrm{RT})$ at $200 \mathrm{~nm}, 0.74 \mathrm{eV}$ for the band half-width $W_{1 / 2}^{C}$, the values of $N$ (in mole ppm) 
ranged between 0.14 and 1.91 for $\mathrm{Bi}^{3+}{ }_{-}$and between 0.19 and 6.74 for $\mathrm{BiO}^{+}$doped $\mathrm{NaCl}$. Similar calculations, performed for $\mathrm{KCl}\left(n \cong 1.719\right.$ and $\left.W_{1 / 2}^{C} \cong 0.87\right)$, yield $N$ equal to 2.56 and $13.63 \mathrm{mppm}$ for the samples studied.

\subsection{Ionic conductivity}

\subsection{1. $\sigma(T)$ plot}

Figure 4 shows the temperature dependence of conductivity $\sigma$ of $\mathrm{NaCl}$ and $\mathrm{KCl}$ crystals doped with $\mathrm{BiO}(+)$ and $\mathrm{Bi}(3+)$, respectively. For the sake of comparison the $\sigma T$ vs. $(1 / T)$ plots for nominally pure (with few ppm of divalent impurities) $\mathrm{NaCl}$ and $\mathrm{KCl}$ as well as for $\mathrm{KCl}: \mathrm{Pb}(2+)$ crystals are also presented. These dependencies display two weakly expressed regions, the extrinsic and the association one. Similar to $\mathrm{Me}^{2+}$ ions, the larger the dopant concentration, the higher the temperature at which association with vacancies occurs. Estimated values of the association energy are nearly equal to $0.68 \mathrm{eV}$, which differs considerably from the value characteristic of divalent impurities, cp. $[12,13]$.

\subsubsection{Solubility of bismuth and the excess charge compensation}

According to Stoicescu et al. [14] it is not easy to obtain the alkali halide crystals with a large amount of trivalent cations. This fact is in contrast to the situation in AH: $\mathrm{Me}^{2+}$ crystals for which the dopant solubility increases with decreasing ionic radius of the impurity. It seems reasonably to suppose that for the trivalent cations the increasing difference in valency between the substituted and the substituting cations yields a decrease in both the solubility limit of the dopant and the stability of the solid solution formed. Although the co-presence of some aliovalent anions should affect both mentioned quantities, the effect of $\mathrm{O}^{2-}$ inferred from the absorption data, for instance, is not clear from the plots of electrical conductivity. It can be seen, for instance, that for the $\mathrm{BiO}^{+}$doped $\mathrm{NaCl}$ crystals the conductivity is practically independent of the presence of the dopant. When - similar to silver halides [5] — bismuth ions enter the lattice of alkali halides substitutionally, each impurity ion should be accompanied by two cation vacancies. Then, neglecting the number of intrinsic defects, the conductivity characteristic of the association region should be for trivalent cations twice as large as for the divalent ones. Moreover, the association with cation vacancies should start at higher temperatures and the electrostatic interactions should be stronger in the case of $\mathrm{Me}^{3+}$ doped alkali halides than for the $\mathrm{Me}^{2+}$ doped crystals for which only one cation vacancy is involved. Although some of these phenomena were detected experimentally, see Fig. 3, low solubility of bismuth makes the quantitative analysis of the obtained results difficult. Among others, the problem related with the number of excess charge compensating vacancies remains open. The difference in the association energy values, related to di- and trivalent cations, could be understood if one assumes that only one cation vacancy is tightly bounded with the trivalent cation. The second vacancy should be located at a position further than the next nearest neighbour one and bound with much less energy. For this and other reasons, it seems necessary to compare data obtained for di- and trivalent cations of chemically the same element. 

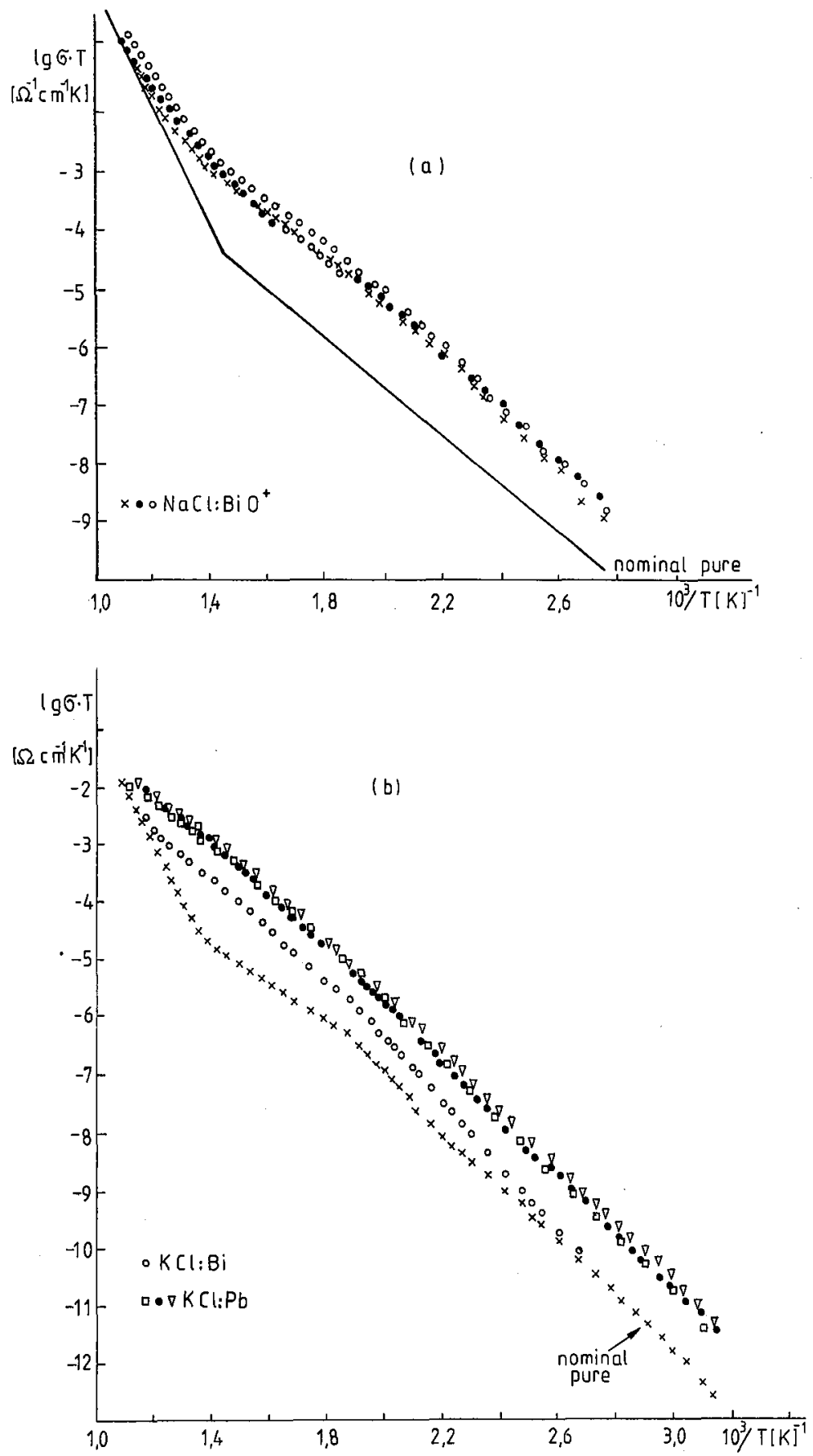

Fig. 4. Temperature dependence of electric conductivity of $E$ (a) and $M(b)$ crystals; in (b) the data characteristic of $\mathrm{KCl}: \mathrm{Pb}(2+)$, according to [12], are also presented. 


\subsection{ITC data}

\subsubsection{AR state}

For plate-shaped AR samples at least one low- $(A)$ and one high-temperature $(C)$ ITC band were observed; Figs. 5-8 show some examples of the data obtained. The low-temperature band is already present in AR samples, and its amplitude $H^{A}$ reacts to the polarizing field $E_{\mathrm{p}}$ in a way similar to the dipole band in $\mathrm{AH}: \mathrm{Me}^{2+}$ systems, e.g. [10]. For $\mathrm{KCl}: \mathrm{Bi}^{3+}$ crystals the band also reacts to the polarizing temperature $T_{\mathrm{p}}$ resembling the behaviour of the low-temperature band in the LiF: $\mathrm{Ti}^{3+}$ system [1]. Moreover, in all cases the $A$ band is positioned below the dipole band characteristic of divalent impurities in alkali halides. The linearity of the $H^{A}\left(E_{\mathrm{p}}\right)$ dependence and relatively weak polarizations related with this band are consistent with the main features of dielectric relaxations induced by low concentrations of non-interacting dipolar defects. Although the geometrical model of such defects is not univocally known for trivalent cations, the concept of I.V.V.
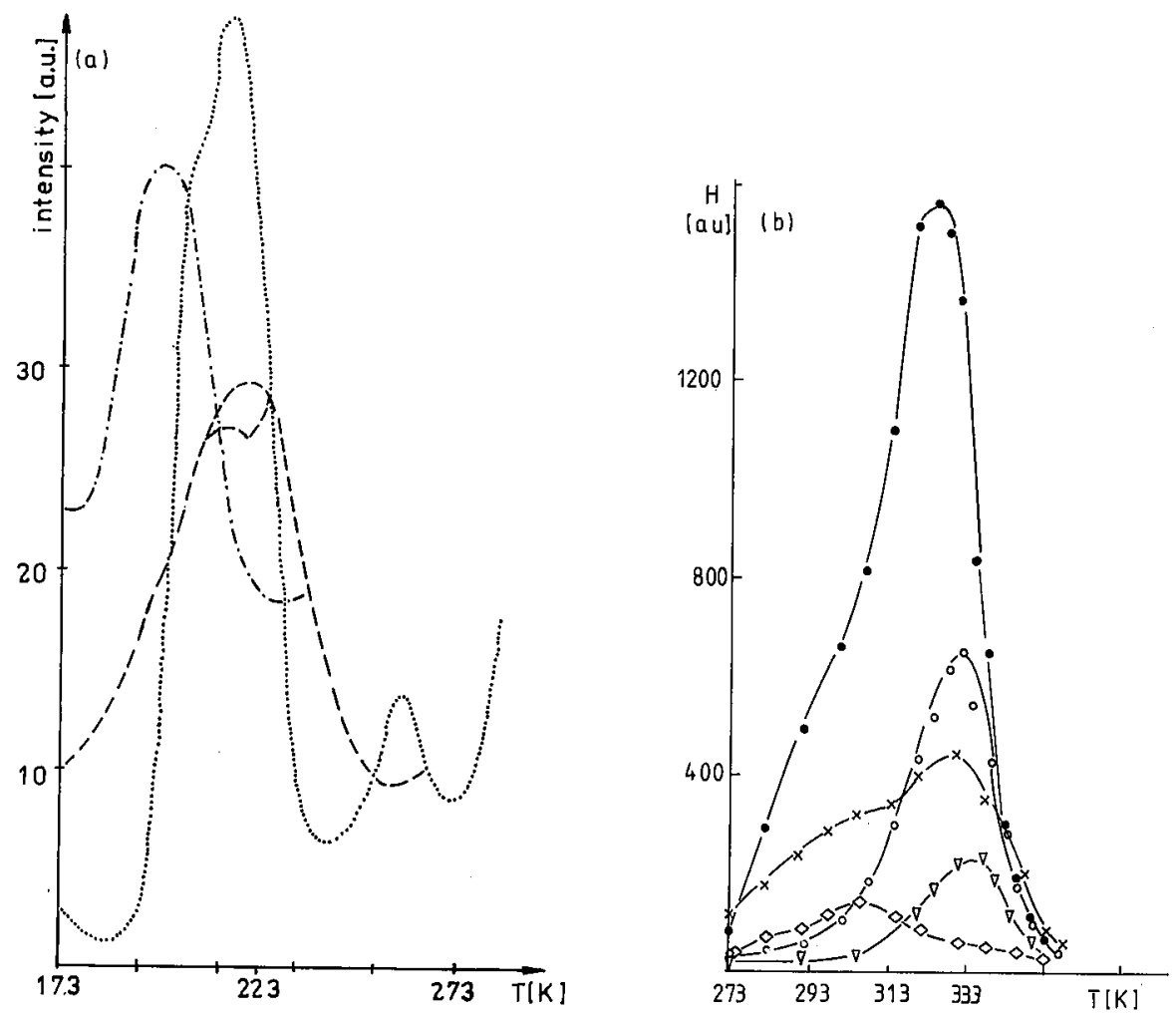

Fig. 5. ITC of crystals in the (a) low- and (b) high-temperature range; polarization conditions for all samples: $T_{\mathrm{p}} \cong \mathrm{RT}, T_{\mathrm{f}}=178 \mathrm{~K}, V_{\mathrm{p}}=10^{3} \mathrm{~V}$. Spectra of the following

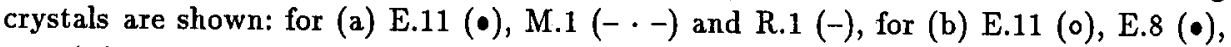
M.1 (ם), R.1 ( $\nabla)$ and D.2 (x). 

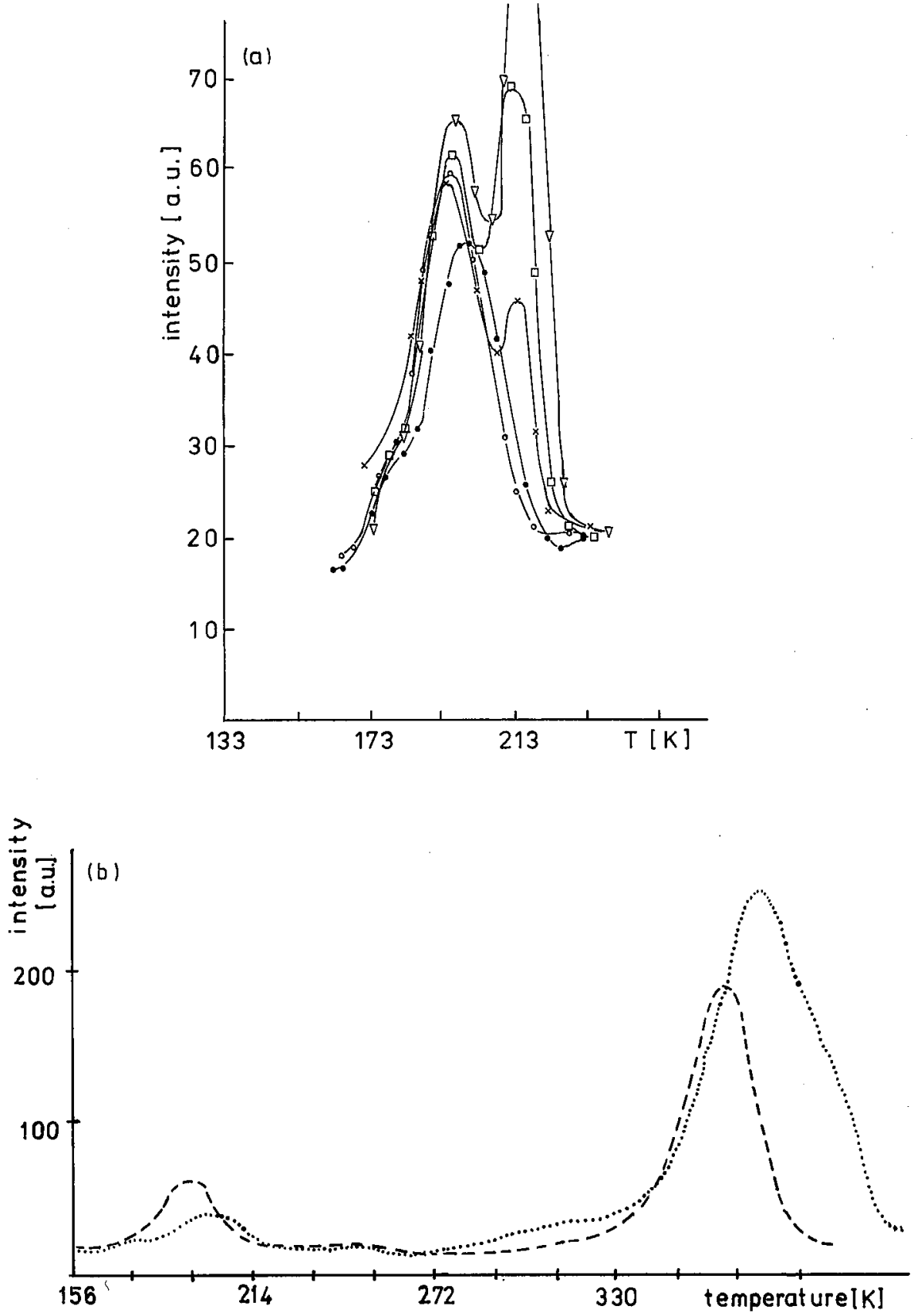

Fig. 6. The effect of solution treatment and RT ageing for M.1 (a) and M.2 (b) crystals; $T_{\mathrm{p}} \cong \mathrm{RT}, T_{\mathrm{f}}=178 \mathrm{~K}, V_{\mathrm{p}}=10^{3} \mathrm{~V}$. The bands were obtained for AR $(\bullet)$, ST $(\nabla)$ and RT stored samples $(\square, \times, \circ,-)$. 

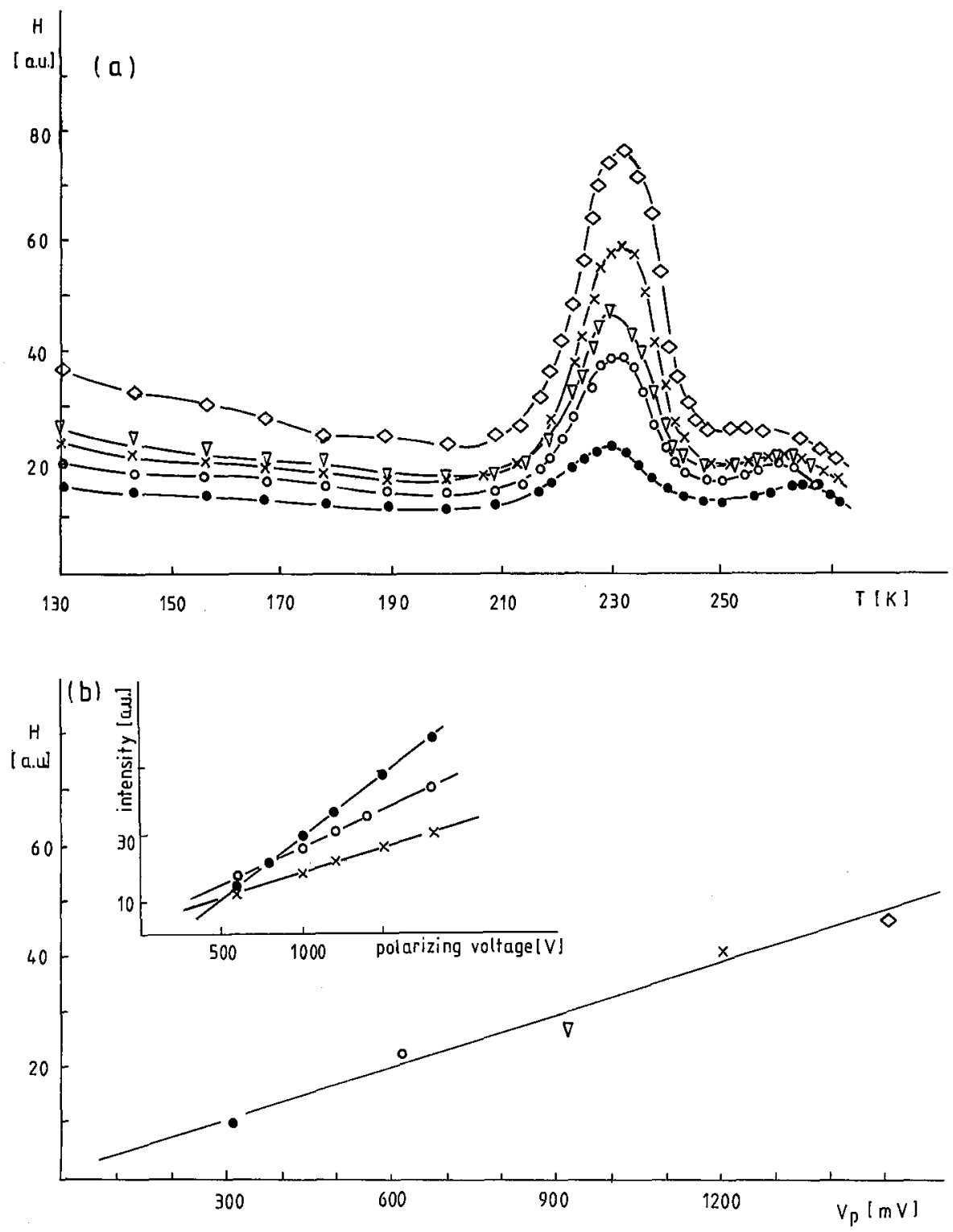

Fig. 7. Effect of $V_{\mathrm{p}}$ upon the dipolar band (a) and the relationship between $I I$ and $V_{\mathrm{p}}$ (b) for R.1; $T_{\mathrm{p}}=228 \mathrm{~K}, T_{\mathrm{f}}=209 \mathrm{~K}$. The inset of (b) shows the $H\left(T_{\mathrm{p}}\right)$ plots for E. $6(\bullet)$, M.1 $(0)$ and D.2 $(\triangle)$ crystals; in this case $T_{\mathrm{p}} \cong \mathrm{RT}, T_{\mathrm{f}}=178 \mathrm{~K}$.

dipoles satisfactorily accounts for the experimental observations. Some differences - with respect to the $\mathrm{AH}: \mathrm{Me}^{2+}$ crystal systems - were also detected in the high-temperature region. For instance, no simple concentration dependence was observed neither for the amplitude of the high-temperature band $H^{C}$ nor for the 


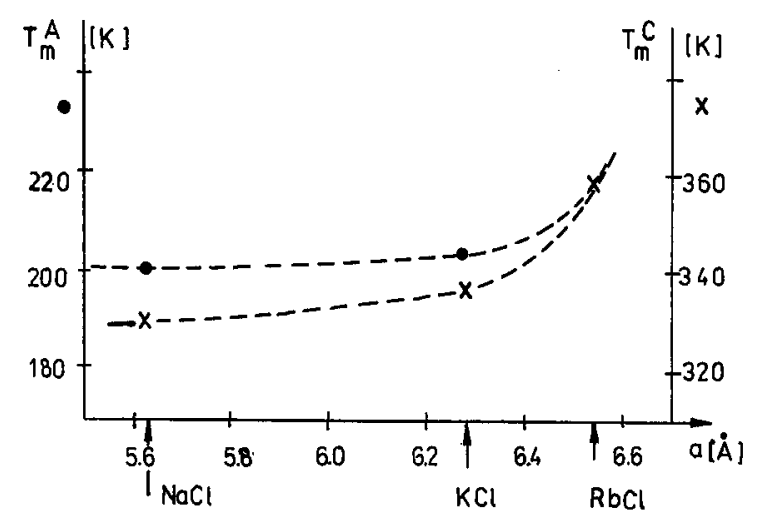

Fig. 8. Empirical relation between the peak positions of the low- $\left(T_{m}^{A}\right)$ and high- $\left(T_{\mathrm{m}}^{C}\right)$-temperature bands and the lattice parameter $a$ of $\mathrm{NaCl}, \mathrm{KCl}$ and $\mathrm{RbCl}$ crystals.

position of its maximum $T_{\mathrm{m}}^{C}$; on the other hand, the width of this band increases with the increasing content of bismuth.

\subsubsection{Effecls related with the solution treatment and plastic deformation}

Water affinity of trivalent bismuth is responsible for the presence of some quantity of $\mathrm{BiO}^{+}$ions in freshly cutted $\mathrm{NaCl}$ samples annealed in air at high temperatures. This results in strong overlapping of the low-temperature band with relaxations of defects containing the oxygen-like ions.

Prolonged annealing (up to about $3.5 \mathrm{~h}$ ) at $873 \mathrm{~K}$ of $\mathrm{RbCl}: \mathrm{Bi}^{3+}$ samples results in the increase in the low-temperature band at the expenses of the high-temperature one. Such conversion of bands was not detected previously, neither for $\mathrm{Me}^{2+}$ doped alkali halides [10] nor for $\mathrm{Me}^{3+}$ doped silver halides [5]. Because some of the mixed bismuth-potassium salts are chemically very stable, the re-solution of precipitated phases present in the $\mathrm{AR} \mathrm{KCl}: \mathrm{Bi}^{3+}$ samples is not a simple task. It was stated, for instance, that the low-temperature ITC band remains unchanged after prolonged $(3.5 \mathrm{~h})$ high-temperature annealing. A peculiar reaction of the considered relaxations was observed for plastically deformed samples. For example, compression of the $\mathrm{BiO}^{+}$doped $\mathrm{NaCl}$ crystals results in the appearance of an additional low-temperature band positioned below the main dipolar one. Its amplitude $H$ decreases with the storage at RT, and $48 \mathrm{~h}$ is enough for complete disappearance of this band. On the other hand, few percent of compression induces strong decrease in the high-temperature band amplitude, a distinct shift of its position towards higher values and a drastic increase in the bandwidth. Although some of these phenomena resemble the behaviour of the $C$ band for $\mathrm{AH}: \mathrm{Me}^{2+}$ systems, interpretation of the obtained results cannot be done in terms of polarizations related with the classical Cottrell atmosphere. In contrast to divalent ions, whose ionic radius is comparable with that of the matrix, the small trivalent impurities are segregated near dislocations probably in the form of aggregates and/or precip- 
itates which transform into simpler defects during both thermal and mechanical treatment of samples.

\section{Summary}

1. Optical absorption spectra of $\mathrm{Bi}^{3+}$ ions are similar to those typical of other heavy-metal ions with the $s^{2}$ electronic configuration; hence, the absorption can be exploited for analytical purposes.

2. Strong water affinity of trivalent bismuth and preferential bonding between $\mathrm{Bi}^{3+}$ and $\mathrm{O}^{2-}$ ions are responsible for the formation of $\mathrm{BiO}^{+}$molecular ions under conditions of crystal growth and during the high-temperature annealing of samples.

3. Exceptionally small solubility of the dopant can be observed, among others, in small differences of conductivity between the doped and nominally pure samples.

4. The charge compensating elements (either cation vacancies or the oxygen-like ions) together with trivalent bismuth give centres endowed with electrical moment; the reorientation of these "dipoles" can be monitored by the ITC technique.

5 . The composed character of the impurity related defects is reflected in the complexity of all ITC spectra observed.

\section{References}

[1] R. Capelletti, M.G. Bridelli, M. Friggeri, G. Ruani, in: Proc. 5th Symp. Electrets, IEEE, Heidelberg (Germany) 1985, IEEE, New York 1985, p. 294.

[2] M. Hartmanova, E. Mariani, M. Lebl, Czech. J. Phys. B 22, 623 (1972).

[3] M. Hartmanova, M. Lebl, E. Mariani, Cryst. Lattice Defects 4, 287 (1973).

[1] S. Radhakrishna, A.M. Karguppikar, J. Phys. Chem. Solids 34, 1497 (1973).

[5] I. Kunze, P. Müller, Phys. Status Solidi 38, 271 (1970).

[6] R. Voszka, I. Tarjan, L. Berkes, J. Krajsovszky, Krist. Technik 1, 423 (1966).

[7] R. Fleischer, Acta Metal. 9, 996 (1961).

[8] E. Orowan, in: Proc. Symp. Internal Stresses in Metals and Alloys, University Press, London 1948, p. 451.

[9] C. Bucci, R. Fieschi, G. Guidi, Phys. Rev. 148, 816 (1966).

[10] M. Suszyńska, R. Capelletti, Cryst. Res. Technol. 19, 1489 (1984).

[11] A. Fukuda, Sci. Light (Tokyo) 13, 64 (1964).

[12] M. Suszyńska, M. Hartmanova, H. Rezabkova, M. Lebl, Czech. J. Phys. B 26, 1011 (1976).

[13] F. Beniere, R. Rokbani, J. Phys. Chem. Solids 36, 1151 (1975).

[14] Gh. Stoicescu, S.V. Nistor, C.D. Mateescu, Phys. Status Solidi B 156, 411 (1989). 